\title{
Guidance for catheter ablation of ventricular arrhythmia
}

\author{
Mark Potse $\cdot$ Vidal Essebag
}

Received: 19 November 2008/Accepted: 9 December 2008/Published online: 10 January 2009

(C) The Author(s) 2008. This article is published with open access at Springerlink.com

Efficient pumping of the heart requires simultaneous contraction of all cardiac muscle cells. The necessary coordination is obtained by transmission of "action potentials:" periodic changes in transmembrane voltage which, in muscle cells, trigger the calcium influx needed for contraction. In cardiac muscle, they also cause an electric current flow to neighboring cells through intercellular connections. This current causes an action potential in each neighbor, leading to rapid activation of the whole heart. Normally the initial activation comes from a specialized region called the sinus node, at a regular pace adapted to the needs of the body. Ventricular tachycardia (VT) is a potentially fatal disturbance of this mechanism, characterized by a very fast heart rate and an abnormal activation pattern.

VT can be caused by an "ectopic focus," a small area of tissue that starts activating itself and its neighbors on its own account. A far more common cause, however, are scars due to infarction, cardiomyopathy, or surgery. These can embed a maze of small surviving muscle fibers through which activation may proceed during a substantial part of a heartbeat before it reaches the healthy myocardium again. The activation may then round the scar and re-enter the narrow pathway (see Fig. 1). Continuous reentry in this fashion results in re-entrant VT.

\footnotetext{
M. Potse ( $\square)$

Research Center, Sacré-Coeur hospital, Montreal, QC, Canada e-mail: mark@potse.nl

M. Potse

Laboratory for Experimental Cardiology,

Academic Medical Center, Amsterdam, The Netherlands

V. Essebag

Division of Cardiology, Sacré-Coeur Hospital and McGill

University Health Center, Montreal, QC, Canada
}

Treatment options for VT include pharmacological suppression, implantation of a cardioverter-defibrillator (ICD), surgical resection of the arrhythmogenic area, and catheter ablation. None of these methods is perfect. Pharmacological treatment often fails [7]. An ICD can reliably terminate VT but does not prevent it from recurring; frequent ICD shocks can cause significant morbidity [7]. Surgical treatment is limited by its invasiveness and relatively high morbidity and mortality. Catheter ablation is only minimally invasive and, if successful, can provide a definitive cure. It is presently considered as a first-choice treatment for focal VT in patients with structurally normal hearts, and as a secondary treatment in patients who are experiencing too many ICD discharges.

Procedures to find a suitable ablation site include "pace mapping" and "activation sequence mapping." In pace mapping, the heart is stimulated from the catheter tip, resulting in an electrocardiogram (ECG) that mimics the ECG that would be obtained if the VT originated at the catheter tip location. Comparison with an ECG recorded during VT provides an estimate of the origin relative to the catheter tip. After displacing the catheter and stimulating again, a new ECG is made. This procedure is repeated until the two ECGs match, and allows for the localization of the "exit site," where the abnormal activation reaches the healthy myocardium. Patients with more than one VT circuit can have several exit sites.

In activation sequence mapping, the VT is induced, and the activation pattern is assessed by recording signals at many locations with one or two catheters. The goal is to find the earliest activated site. This is a time-consuming procedure, during which the patient has to be kept in VT. To gain time, the exit site found by pace mapping can be used as a starting point [3]. 

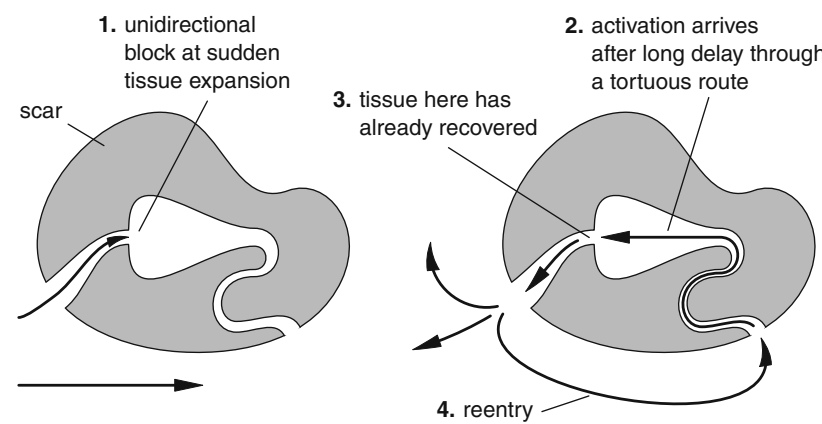

Fig. 1 The concept of reentrant tachycardia due to an infarction scar (gray). Left Activation (arrows) comes from the left, enters a narrow surviving pathway, and blocks at a point where the pathway suddenly expands. Right Activation arriving from the other side, delayed due to a long and tortuous route, can re-activate the first pathway and the bulk myocardium, and initiate a reentry

When catheter ablation became mainstream, in the early 1990s, difficulties encountered included the need for radiography to find out where the catheter was, and the need to rapidly infer from a standard 12-lead ECG where it should be going. Several attempts have been made to facilitate procedures with the help of electrocardiographic body surface maps (BSMs) and computer interpretation of the recorded signals. The earliest attempts were to boldly use inverse models to estimate the exit site from the BSM measured during VT. A suitable model for this purpose is the single moving dipole [5]. This required an accurate anatomic model of the patient and a high-quality BSM, while still having error margins in the order of centimeters.

Subsequent attempts were more pragmatic. Atlases of paced BSMs were created, which linked potential patterns to segments of the myocardium $[1,3]$. This approach had a resolution similar to that of inverse models, but it was much faster and did not require an accurate anatomic model. An algorithm that interpolated in such databases could generate accurate catheter displacement advice [4].

A different pragmatic approach is described by Fukuoka et al. in this issue of MBEC [2]. Their idea is to use a moving-dipole model, but now without accurately modeling the patient's anatomy. Absolute errors of this method are very large. The trick that makes it useful is to use the same method to also estimate the position of the catheter tip. For this purpose a current dipole is produced by the electrodes on the catheter. This results in large errors for the two positions themselves, but a reasonable estimate of the exit-site position relative to the catheter [2]. The advantage with respect to database interpolation [4] is that no pacing is required to bring the catheter to the exit site. This could make the procedure considerably shorter.

However, the application of all BSM-based methods has been limited because cardiologists were not eager to apply the required 60 or more surface electrodes on their patients during an ablation procedure. Moreover, two recent technical developments revolutionized catheter ablation procedures in a very different way. Catheter localization systems based on electric and magnetic fields can now track the catheter position in real-time, without radiography, and with high accuracy [6]. This made both pace mapping and activation sequence mapping much easier. In addition, noncontact catheters were introduced, which can estimate the activation sequence of the ventricles from a single heartbeat.

These two developments changed particularly the way in which infarct-related VTs were investigated. Assisted by accurate real-time catheter localization, cardiologists now record signals during normal cardiac rhythm to locate the infarct scar. Such "substrate mapping" allows unstable VTs to be addressed that could not be located with activation mapping [6]. In addition, they search for spurious "pre-diastolic" activations or "late potentials," which can indicate the narrow pathways that give rise to VT, even when the VT is not happening. Accurate maps of the circuits can be made, and a "critical isthmus" can be identified and targeted for ablation. Exit sites have become less interesting, and clinical testing of BSM-based procedures has been abandoned.

This does not have to remain so forever. The word "pendulum" is often used when developments in medicine are described. More than once, technology has changed the way things were done in the electrophysiology laboratory. The development of such technology can take a long time, too long for biomedical engineers to start working only when a pendulum swings the right way. From this perspective, Fukuoka et al. must be congratulated for the perseverance with which they continued to develop and test the method which they first proposed some 8 years ago.

Two concepts that must be well distinguished in the paper by Fukuoka et al. [2] are the exit site and the target site for ablation. These may coincide in focal VT, but they are generally distinct when a scar is involved. Methods based on the surface ECG or BSM can only guide the catheter to the exit site. From there, other methods have to be used to find a suitable ablation site. It is tempting to consider ablation of the exit site itself when a VT is not mappable. Although this is presently not the strategy of choice, the idea to use it as an alternative to long linear and circumferential lesions in case of unmappable VT deserves to be considered.

Much remains to be done. As Fukuoka et al. mentioned, their method has to be tested in real patients. Clinical partners have to be found. This could be easier with a smaller electrode set. Computer-assisted VT localization methods distinguish themselves from the traditional methods by the use of many electrodes and, as a consequence, by the use of quantitative analysis. How 
well quantitative analysis works with respect to VT localization with a limited electrode set has not been investigated yet.

In conclusion, technology that can make VT localization faster or more reliable may help to turn more "unmappable" VTs into mappable ones_-and cure more patients.

Open Access This article is distributed under the terms of the Creative Commons Attribution Noncommercial License which permits any noncommercial use, distribution, and reproduction in any medium, provided the original author(s) and source are credited.

\section{References}

1. Dubuc M, Nadeau R, Tremblay G, Kus T, Molin F, Savard P (1993) Pace mapping using body surface potential maps to guide catheter ablation of accessory pathways in patients with WolffParkinson-White syndrome. Circulation 87:135-143
2. Fukuoka Y, Oostendorp TF, Armoundas AA (2009) Method for guiding the ablation catheter to the ablation site: a simulation and experimental study. Med Biol Eng Comput (this issue)

3. Peeters HAP, SippensGroenewegen A, Wever EFD, Ramanna H, Linnenbank AC, Potse M, Grimbergen CA, van Hemel NM, Hauer RNW, Robles de Medina EO (1999) Clinical application of an integrated 3-phase mapping technique for localization of the site of origin of idiopathic ventricular tachycardia. Circulation 99:1300 1311

4. Potse M, Linnenbank AC, Peeters HAP, SippensGroenewegen A, Grimbergen CA (2000) Continuous localization of cardiac activation sites using a database of multichannel ECG recordings. IEEE Trans Biomed Eng 47(5):682-689

5. Savard P, Roberge FA, Perry JB, Nadeau RA (1980) Representation of cardiac electrical activity by a moving dipole for normal and ectopic beats in the intact dog. Circ Res 46:415-425

6. Stevenson WG, Soejima K (2007) Catheter ablation for ventricular tachycardia. Circulation 115:2750-2760

7. Tung R, Zimetbaum P, Josephson ME (2008) A critical appraisal of implantable cardioverter-defibrillator therapy for the prevention of sudden cardiac death. J Am Coll Cardiol 52:1111-1121 